\title{
ICMEg - the Integrated Computational Materials Engineering expert group - a new European coordination action
}

\author{
Georg J Schmitz ${ }^{1}$ and Ulrich Prahl $2^{*}$
}

\author{
* Correspondence: \\ Ulrich.Prahl@iehk.rwth-aachen.de \\ 2Deparment of Ferrous Metallurgy \\ at RWTH Aachen University, Aachen, \\ Germany \\ Full list of author information is \\ available at the end of the article
}

\begin{abstract}
Integrated Computational Materials Engineering, or ICME, applies a range of software tools in simultaneous or consecutive combinations. Major successful developments in the area of ICME up to the present have been essentially driven by academic and industrial users of simulation software. Starting with a brief overview of the benefits provided by ICME, the present article will give a short outline of the ICMEg consortium, the Integrated Computational Materials Engineering expert group, and the related European project. The vision and mission of this project is defined and its practical implementation detailed. The chief aim of ICMEg is to set up a global and open communication standard and protocols on the component scale, on the microstructural scale, as well as basic underlying thermodynamic models and discrete models on the electronic, atomistic and mesoscopic scales.
\end{abstract}

Keywords: Integrated Computational Materials Engineering; ICME; Standardization; Platform concepts; Processing/microstructure/property relationships

\section{Background}

Materials constitute a key enabling technology, allowing products and industrial processes to become more competitive and sustainable. New, knowledge-based materials with engineered properties allow new products and processes. The most relevant research into the rational design of materials is presently being carried out in the fields of 3D-Materials Science (3D-MS), by the Materials Genome Initiative (MGI) and in Integrated Computational Materials Engineering (ICME), all of which are strongly interrelated [1].

"Integrated computational materials engineering as an emerging discipline aiming to integrate computational materials science tools into a holistic system will accelerate materials development, transform the engineering design optimization process, and unify design and manufacturing" [2]. Materials and component properties go through an evolution and depend not only on the process as a whole and the component's load history, but also on component shape and actual alloy composition. The focus of ICME is thus on engineering the properties of a component as a function of the local properties of the material within the component and its manufacturing history.

ICME is generally based on multiscale modeling approaches extending right through the entire manufacturing cycle. Up to the present, related models on the various length 
scales of the component and microstructure as well as along the process chain have evolved at different rates of innovation. The combination of such models nowadays provides the key to describing and controlling microstructural evolution and eventually to engineering effective properties of technical materials and products [3].

The capability of current software tools for materials and process simulation, coupled with the computational power currently available, make efforts towards the integration of these tools possible, meaningful and timely. ICME is a new and powerful discipline, and coupling software tools is still in its infancy. When applying the ICME approach, this kind of coupling presently requires a significant effort in terms of time and manpower, e. g. with regard to data conversion.

Similar to the successful data standards currently under development and maintained by the "joint photographers expert group" (.jpeg/.jpg formats) or the "movie pictures expert group" (.mpeg/.mpg formats), the Integrated Computational Materials Engineering expert group, ICMEg, was recently set up. The aim of this expert group is to define an open communication standard which can be applied for all tools relevant to ICME.

\section{Approach}

\section{ICMEg: vision, mission and consortium}

The Vision of ICMEg is a new strategy of materials and process development in which a range of academic and commercial simulation tools, both present and future, can be easily combined across a range of different process steps, and which span a range of different length scales in a "plug \& play" type architecture based on object-oriented, standardized data exchange (Figure 1).

Once established, an open and easily accessible formulation of a global standard for data exchange in ICME settings will:

- Significantly facilitate the exchange of data between different tools

- Create new options and functionalities for the present tools

- Allow for easy integration between commercial and academic approaches

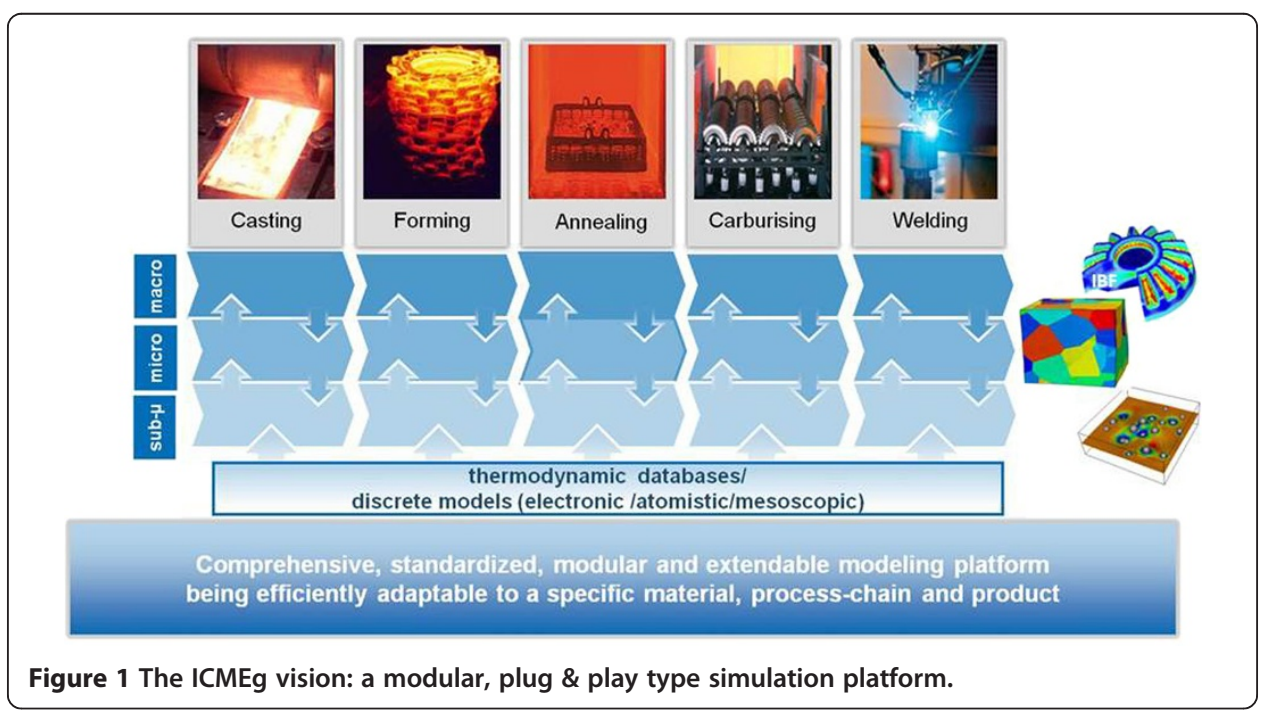


- Provide the pathway for life-cycle modeling of components/products

- Allow for global optimization of process chains

- Stimulate many further new developments

\section{The mission}

ICMEg aims to build up a scientific network of stakeholders interested in incorporating ICME into industrial applications. The stakeholders will benefit from sharing knowledge and best practice. This will promote a deeper understanding between the various communities of materials scientists, production engineers, IT engineers and industrial users.

The first objective of ICMEg is to set up and maintain a network of contacts including:

- Simulation software providers around the world

- Governmental and international standardization authorities

- ICME type users of simulation software

- Various associations in the area of materials and processing

- Academic developers of simulation software

The second objective of ICMEg then is to:

- Stimulate knowledge-sharing in the field of multiscale materials design

- Define an ICME language in the form of an open and standardized communication protocol

- Communicate this standard worldwide to achieve its wide acceptance

- Discuss and decide about future amendments to the initial standard

- Set up a legal body for sustainable further development

\section{The consortium}

ICMEg is open to all who are willing and able to contribute. The initial nucleus for ICMEg is the ICMEg consortium, funded by the European Commission. It comprises eleven partners from six European countries (Figure 2).

The partners are academic institutions, SME and private non-profit-making software providers from the areas of computational materials modeling and simulation of

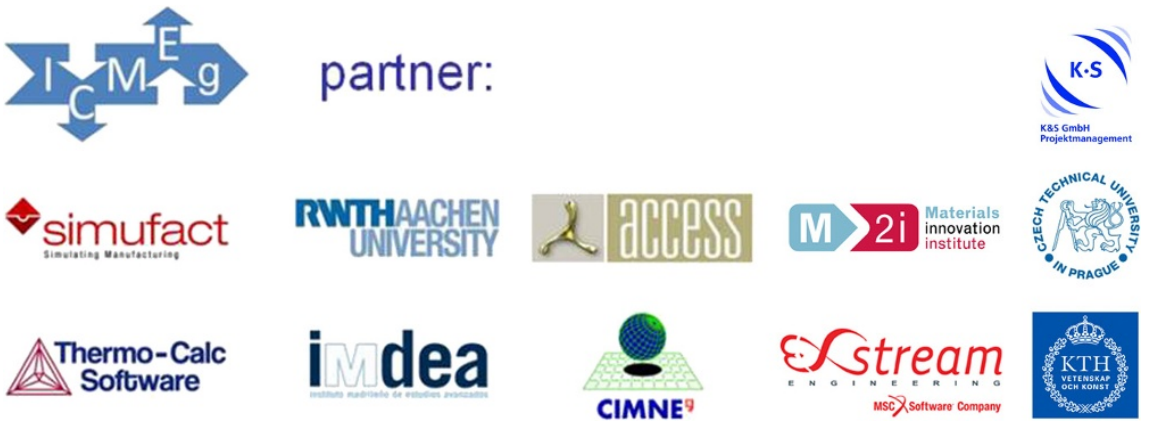

Figure 2 Consortium of the ICMEg project. 
materials processing. Each of them has already initiated individually its own platform strategies towards ICME. To underline the global approach taken by ICMEg, the consortium closely interacts with a number of associated partners around the globe, especially from the US, Japan and India.

The majority of the consortium partners have their origins in structural metallic materials, and some partners contribute expertise regarding other structural materials, such as polymers, composites or concrete. The standard formulations - with the overall aim of being generic - will thus be elaborated on the basis of examples of structural metallic components. Contacts to simulation platform activities in the area of functional materials, coatings, thin films and others have already been partially established and will be fostered within the scope of the ICMEg project.

\section{Outlook - the ICMEg roadmap}

The European ICMEg project was officially launched in early October 2013. Within a three-year period, the ICMEg consortium is aiming to

- organze two international workshops

- edit two books with the following working titles:

1. Directory of Software Providers for ICME

2. Software Standards for Successful ICME

- specify standards for data exchange in ICME settings

- find an association of software providers for ICME

The ICMEg consortium will inform, invite and network researchers and engineers from various communities who are able to contribute to the discussions. Spreading information about ICMEg activities will be done via mailing lists, at conferences, via websites and social media, via publications such as the present article, and other appropriate information channels.

The first workshop is essentially meant to create and establish an initial network of providers of software solutions for ICME. The major result of this first workshop will be a comprehensive and thematically structured overview of existing models, solutions and stakeholders in the field, and will be published as a book.

The major aim of ICMEg is to generate a global and open standard for data exchange in ICME settings. However, the expertise of a single institution cannot cover all the aspects which need to be considered in defining appropriate standard formulations for the heterogeneous range of models and tools. A collective and global effort from a range of different communities is thus indispensible.

This collective effort will be initiated and moderated going forward by the ICMEg consortium. With respect to the collective discussion, a forum will be established and maintained on the ICMEg website (www.icmeg.eu). The discussions in this forum will be stimulated and moderated by the members of the ICMEg consortium, its international advisory committee and other volunteers.

The results of all the respective discussions (within the scope of a full consensus process which is open to all interested and qualified parties) are eventually expected to evolve into a de-facto standard agreed on by a large number of stakeholders. Initial standard formulations will be discussed and reviewed in the second workshop and 
documented in a second book. The association to be founded will ensure sustainable further development of the initial standard formulations after conclusion of the ICMEg project. The de-facto standard is intended to be transformed at a later stage into a formal legal standard with the involvement of international standardization bodies, such as RDA, CODATA, NISO, ASTM and ASM International.

Clearly, the vision of ICMEg is only achievable by a global, collective and collaborative effort. This effort not only has to integrate models and tools, but especially individual players. All those willing and able are cordially invited and strongly encouraged to join in and support this global effort [4].

Competing interests

The authors declare that they have no competing interests.

Authors' contributions

GJS and UP are leading the ICMEg consortium. They jointly discussed and composed the present article. Both authors read and approved the final manuscript.

\section{Acknowledgement}

The ICMEg proposal is based on results of the Cluster of Excellence's "Integrative Production Technologies for High Wage Countries" funded by the Deutsche Forschungsgemeinschaft. The ICMEg project is presently funded by the European Commission under Grant NMP3-CA-2013-606711.

\section{Author details}

${ }^{1}$ Access e.V. at the RWTH Aachen, Aachen, Germany. ${ }^{2}$ Deparment of Ferrous Metallurgy at RWTH Aachen University, Aachen, Germany.

Received: 30 September 2013 Accepted: 20 January 2014

Published: 24 February 2014

References

1. Li M, Campell C, Thornton K, Holm E, Gumbsch P (2013) Proceedings of the 2nd World Congress on Integrated Computational Materials Engineering. Salt Lake City, USA, pp 7-11.7

2. National Research Council: Integrated Computational Materials Engineering (2008) A Transformational Discipline for Improved Competitiveness and National Security. National Academic Press, Washington, D. C. ISBN: 0-309-12000-4

3. Schmitz GJ, Prahl U (2012) Integrative Computational Materials Engineering- Concepts and applications of a modular simulation platform Wiley VCH Verlag Weinheim. ISBN: 978-3-527-33081-2

4. Schmitz GJ, Prahl U (2012) "Integrated Computational Materials Engineering expert group". website www.ICMEg.eu (last access Feb $1^{\text {st }}$ 2014)

doi:10.1186/2193-9772-3-2

Cite this article as: Schmitz and Prahl: ICMEg - the Integrated Computational Materials Engineering expert group - a new European coordination action. Integrating Materials and Manufacturing Innovation 2014 3:2.

\section{Submit your manuscript to a SpringerOpen ${ }^{\circ}$ journal and benefit from:}

- Convenient online submission

- Rigorous peer review

- Immediate publication on acceptance

- Open access: articles freely available online

- High visibility within the field

- Retaining the copyright to your article

Submit your next manuscript at $>$ springeropen.com 